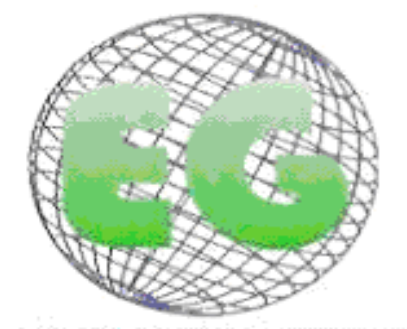

ISSN 1695-6141

N 27

\title{
Prevalencia de los diagnósticos de enfermería en niños que reciben tratamiento oncológico
}

Prevalence of nursing diagnoses in children receiving oncologic treatment

\section{"Rodríguez Quecho, M., "*Rincón Rodríguez, YZ., ***Reyes González, A., ${ }^{* * * *}$ Fajardo Peña, MT,. ${ }^{* * * * *}$ Orozco Vargas, LC., ${ }^{* * * * *}$ Camargo Figuera, FA.}

\begin{abstract}
*Enfermera. Fundación Oftalmológica de Santander Carlos Ardila Lule FOSCAL, UCI Neonatal Clínica Metropolitana de Bucaramanga. Colombia. **Enfermera. Maestría en Urgencias y Emergencias. Residencia para adultos mayores Mediterránea, Gandía-Valencia, España. ***Enfermera. Coordinadora de Enfermería UCI Neonatal Clínica Metropolitana de Bucaramanga.****Enfermera. Especialista en Enfermería Perinatal. Magister en Enfermería. Directora de la Escuela de Enfermería Universidad Industrial de Santander, Bucaramanga-Santander. *****Médico. Profesor Asociado Escuela de Enfermería Universidad Industrial de Santander, Bucaramanga-Santander. ******Enfermero. Magíster en Epidemiología. Profesor Auxiliar Escuela de Enfermería Universidad Industrial de Santander, Bucaramanga-Santander. E-mail: falcamfi@uis.edu.co
\end{abstract}

Palabras clave: prevalencia; diagnóstico de enfermería; enfermería oncológica; enfermería pediátrica. Keywords: Prevalence; Nursing Diagnosis; Oncology Nursing; Pediatric Nursing.

\section{RESUMEN}

El objetivo del presente estudio fue determinar la prevalencia de los diagnósticos de enfermería en niños que reciben tratamiento oncológico.

Se realizó un estudio de corte transversal. Por medio de una entrevista, valoración y aplicación de un formato que permitió establecer los diagnósticos de enfermería presentes en niños en dos instituciones de salud de la ciudad de Bucaramanga, Colombia. Se valoraron 43 niños; 20 niñas $(46.51 \%)$ y 23 niños $(53.49 \%)$, de edades entre los 5 y 13 años.

Los diagnósticos de enfermería más prevalentes fueron: Protección Inefectiva, Riesgo de Infección, Riesgo de Lesión, Náusea y Trastorno de la Imagen Corporal. Los diagnósticos de enfermería con mayor prevalencia están relacionados a efectos del tratamiento oncológico.

El conocimiento de los diagnósticos de enfermería más prevalentes en niños con tratamientos oncológicos permite que el cuidado de enfermería se brinde de acuerdo a estas evidencias. 


\section{ABSTRACT}

The aim of this study was to determine the prevalence of nursing diagnoses in children receiving cancer treatment.

A cross-sectional study was carried out by means of an interview, assessment and implementation of a format that allowed us to establish the nursing diagnoses presented in children from two health institutions in the city of Bucaramanga, Colombia. 43 children, 20 girls (46.51\%) and 23 children (53.49\%) aged between 5 and 13 years old, were evaluated.

The most prevalent nursing diagnoses were: Ineffective protection, risk of infection, risk of injury, nausea and body image disorder. The most prevalent nursing diagnoses are related to effects of the cancer treatment.

The knowledge of the most prevalent nursing diagnoses in children with cancer treatments allows nursing care to be provided according to the evidence.

\section{INTRODUCCIÓN}

El proceso de enfermería se define como un método sistemático y dinámico para establecer un diagnóstico a partir de la valoración del estado de salud, realizar intervenciones según un plan de cuidado y evaluar su efectividad ${ }^{(1)}$. A lo largo de la historia, el proceso de enfermería se ha convertido en una herramienta valiosa para la investigación y el desarrollo de la profesión ${ }^{(2)}$, contribuye al enriquecimiento de la enfermería como ciencia, promueve la autonomía y la utilización de un lenguaje propio de la profesión ${ }^{(3)}$. Y dentro del proceso de enfermería, la valoración y formulación de diagnósticos de enfermería son esenciales para encontrar las necesidades reales o potenciales en una determinada población, facilitando de esta manera intervenciones adecuadas y oportunas.

La enfermera es responsable de la identificación de las respuestas humanas de las personas a su cuidado, es por ello que determinar los diagnósticos de enfermería que se presentan con mayor frecuencia en niños que reciben tratamiento oncológico, tiene como fin facilitar la priorización de las intervenciones y actividades enfocadas a su cuidado, iniciando la elaboración de planes de atención encaminados a mejorar la atención brindada al niño con cáncer y su familia ${ }^{(4)}$.

Adicionalmente, el cáncer ha experimentado un aumento en su frecuencia en los últimos años, por lo cual ha sido reconocido como un importante problema de salud. En el ámbito mundial aproximadamente el $10 \%$ de las muertes en menores de 15 años ocurren por cáncer ${ }^{(5)}$. En Colombia, el Departamento Administrativo Nacional de Estadística (DANE) reportó que el cáncer en niños fue la primera causa de muerte por enfermedad entre los 5 y los 14 años de edad y ocupa el segundo lugar en la mortalidad global en niños después de las muertes violentas ${ }^{(6)}$. Según estimaciones para el actual milenio la incidencia del cáncer en esta población infantil sobrepasará los 250.000 casos por año ${ }^{(7)}$.

Las personas que presentan patologías oncológicas, con frecuencia, comparten las mismas necesidades de cuidado de enfermería; la complejidad de su atención es alta debido a la gran variedad de síntomas físicos, limitaciones funcionales y trastornos psicosociales que se pueden llegar a presentar durante el desarrollo de la enfermedad y tratamiento ${ }^{\left({ }^{(8)}\right.}$.

Actualmente, la oncología infantil representa un desafío para el equipo de salud, especialmente para la enfermera, es así como la formación de especialistas en enfermería oncológica ha permitido un gran avance en los resultados de salud y calidad de vida en los niños ${ }^{(9)}$. Dada la problemática en todos los aspectos de la vida que acarrea esta 
enfermedad y del impacto del diagnóstico oncológico, la enfermera debe realizar una valoración exhaustiva que incluya tanto características clínicas y fisiológicas como psicológicas y mentales que estén presentes debido a la enfermedad o al tratamiento de la misma, además de las implicaciones escolares, sociales y económicas tanto para el niño como para su familia ${ }^{(10)}$.

Siendo la enfermera el profesional de salud responsable del cuidado de esta población, es indispensable que por medio de sus intervenciones logre minimizar y controlar estos efectos negativos que surgen en el transcurso de la enfermedad y durante el tratamiento, y además, se convierta en el apoyo principal del niño y su familia en el proceso. Adicionalmente, no se encontraron en la literatura estudios que mostraran cuál es la frecuencia, incidencia o prevalencia, de los diagnósticos de enfermería en niños con cáncer; por tanto, el objetivo principal del presente estudio es determinar la prevalencia de los diagnósticos de enfermería en niños que reciben tratamiento oncológico.

\section{MATERIALES Y MÉTODOS}

El tipo de estudio que se realizó fue de corte transversal. El estudio se realizó en los servicios de oncología del Hospital Universitario de Santander y de la Clínica Materno Infantil San Luís, ambos de la ciudad de Bucaramanga, Colombia. La recolección de la información se realizó por un periodo de tiempo de dos meses durante el segundo semestre del 2009.

Durante dicho periodo fueron elegidos para participar en el estudio todos los niños y niñas consecutivamente con edades entre los 5 y 13 años y que se encontraran ingresados para recibir tratamiento oncológico en cualquiera de las siguientes modalidades: quimioterapia, radioterapia, cirugía o cuidado paliativo, en las dos instituciones anteriormente mencionadas. No fueron incluidos en el presente estudio niños con estados de conciencia alterados y niños con problemas de comunicación verbal.

Para determinar la presencia de los diagnósticos de enfermería se llevó a cabo en cada participante una valoración focalizada de enfermería del estado de salud, valoración que tenía como fuente de datos el examen físico y la entrevista. Para dicha valoración focalizada se tuvo en cuenta como guía un formato de valoración para la recolección de los datos, el cual se elaboró a partir de dos instrumentos validados anteriormente por la escuela de Enfermería de la Universidad Industrial de Santander.

Mediante una revisión de la Clasificación de Diagnósticos de Enfermería de NANDA-I ${ }^{(11)}$ y las recomendaciones del manual de Carpenito ${ }^{(12)}$ se seleccionaron 27 diagnósticos de enfermería aplicables para la presente investigación y de interés para los investigadores a ser valorados. Para estos 27 diagnósticos de enfermería el formato de valoración guía constaba de una serie de preguntas de tamizaje que sospechan los posibles diagnósticos; posteriormente se evaluó si las características definitorias principales de cada uno de estos diagnósticos estaban o no presentes. El formato valora las posibles respuestas humanas que se presentaban en los niños, debido a la patología y/o al tratamiento que reciben.

Los diagnósticos elegidos junto con sus códigos de identificación de la clasificación NANDA fueron: (00092) Intolerancia a la Actividad, (00007) Hipertermia, (00095) Insomnio, (00111) Retraso en el Crecimiento y Desarrollo, (00002) Desequilibrio Nutricional por Defecto, (00048) Deterioro de la Dentición, (00045) Deterioro de la Mucosa Oral, (00097) Déficit de Actividades Recreativas, (00088) Deterioro de la Deambulación, (00093) Fatiga, (00132) Dolor Agudo, (00133) Dolor Crónico, (00013) Diarrea, (00011) Estreñimiento, (00015) Riesgo de Estreñimiento, (00016) Deterioro de la Eliminación Urinaria, (00134) Náusea, (00004) 
Riesgo de Infección, (00035) Riesgo de Lesión, (00043) Protección Inefectiva, (00120) Baja Autoestima Situacional, (00118) Trastorno de la Imagen Corporal, (00052) Deterioro de la Interacción Social, (00053) Aislamiento Social, (00124) Desesperanza, (00148) Temor, (00046) Deterioro de la Integridad Cutánea.

También fueron evaluadas otras variables para caracterizar la población, como la edad del niño, el género, la procedencia, la escolaridad, el tipo de cuidador principal, el tratamiento recibido y el diagnóstico médico establecido. La recolección de los datos fue realizada por estudiantes de enfermería de último año. La entrevista y valoración requirieron de un tiempo aproximado de 30 minutos durante el cual el niño se encontraba en compañía de los padres y/o cuidadores principales.

El procesamiento de los datos se hizo en el programa Epi Info $6.04 d^{(13)}$ donde fue digitada una base de datos doble e independiente, bases que luego fueron contrastadas y corregidas, para luego ser exportadas para su análisis al paquete estadístico Stata $10^{(14)}$. Se realizó un análisis descriptivo de las características generales de la población de estudio. Las variables cualitativas se presentan con frecuencias absolutas y relativas, para las variables cuantitativas medidas de tendencia central y de dispersión. Para dar cumplimiento al objetivo del estudio se calcularon prevalencias para cada uno de los diagnósticos de enfermería con su respectivo intervalo de confianza del 95\%. También se determinó el número de diagnósticos de enfermería presentados por persona.

Durante la realización de la presente investigación prevaleció el respeto a la dignidad y la protección de los derechos y el bienestar de los niños y las niñas a estudio y los demás principios éticos contemplados en la Resolución 008430 de 1993 para la investigación con seres humanos. Esta investigación es de riesgo mínimo según las categorías contempladas en la resolución antes mencionada, ya que no representó ningún riesgo para el niño o niña participante. Se obtuvo consentimiento informado por escrito del representante legal del niño y el consentimiento verbal del menor; el niño fue incluido a la investigación si ambas partes estaban de acuerdo. El Comité de Ética de la Facultad de Salud de la Universidad Industrial de Santander dio la aprobación al presente estudio con código de registro № IF5109029.

\section{RESULTADOS}

La caracterización en su aspecto sociodemográfico y clínico de los 43 niños participantes del estudio es presentada en la Tabla I. De estos resultados descriptivos se puede resaltar que el género masculino fue el más frecuente, la mayoría tenían una procedencia urbana, los padres eran los cuidadores principales. En cuanto a la enfermedad, las leucemias fueron las patologías más prevalentes y el total de ellos recibían quimioterapia, mientras que menos del $20 \%$ recibían algún otro tipo de tratamiento y la modalidad por la que recibían el tratamiento fue principalmente la hospitalización. Las edades de los niños participantes se encontraron entre 5 y 13 años, con una edad media de 8.9 años ( $D E=2.1$ años). El $50 \%$ de los niños había cursado y aprobado al menos 3 años de escuela primaria (Rango de 0 a 6 años).

En lo relacionado a la prevalencia de los diagnósticos de enfermería, los resultados de la Tabla II muestran que los de mayor prevalencia fueron: Protección Inefectiva, Riesgo de Infección, Riesgo de Lesión, Náusea y Trastorno de la Imagen Corporal, con prevalencias por encima del $50 \%$. Los diagnósticos de enfermería menos frecuentes, con prevalencias por debajo del 5\% fueron: Diarrea, Riesgo de Estreñimiento y Deterioro de la Eliminación Urinaria. Ningún niño presentó el diagnóstico de enfermería Dolor Crónico. 
[Tabla I. Descripción de los participantes de acuerdo a características sociodemográficas y clínicas. Bucaramanga, Colombia.]

\begin{tabular}{|c|c|c|}
\hline Característica & $n=43$ & $\%$ \\
\hline \multicolumn{3}{|l|}{ Genero } \\
\hline Masculino & 23 & 53.5 \\
\hline Femenino & 20 & 46.5 \\
\hline \multicolumn{3}{|l|}{ Procedencia } \\
\hline Urbana & 24 & 55.8 \\
\hline Rural & 19 & 44.2 \\
\hline \multicolumn{3}{|l|}{ Cuidado Principal } \\
\hline Padres & 31 & 72.1 \\
\hline Abuelos & 9 & 20.9 \\
\hline Tíos & 3 & 7.0 \\
\hline \multicolumn{3}{|l|}{ Tipo de Cáncer } \\
\hline Leucemia Linfocítica Aguda & 23 & 53.5 \\
\hline Leucemia Mieloide Aguda & 6 & 14.0 \\
\hline Linfoma no Hodgkin & 4 & 9.3 \\
\hline Meduloblastoma & 4 & 9.3 \\
\hline Neuroblastoma & 2 & 4.6 \\
\hline Otros & 4 & 9.3 \\
\hline \multicolumn{3}{|l|}{ Tratamiento con Quimioterapia } \\
\hline Si & 43 & 100 \\
\hline No & 0 & 0 \\
\hline \multicolumn{3}{|l|}{ Tratamiento con Radioterapia } \\
\hline $\mathbf{S i}$ & 7 & 16.3 \\
\hline No & 36 & 83.7 \\
\hline \multicolumn{3}{|l|}{ Tratamiento con Cirugía } \\
\hline $\mathbf{S i}$ & 2 & 4.6 \\
\hline No & 41 & 95.4 \\
\hline \multicolumn{3}{|l|}{ Tratamiento Alternativo } \\
\hline $\mathbf{S i}$ & 5 & 11.6 \\
\hline No & 38 & 88.4 \\
\hline \multicolumn{3}{|l|}{ Tratamiento Paliativo } \\
\hline $\mathbf{S i}$ & 2 & 4.6 \\
\hline No & 41 & 95.4 \\
\hline \multicolumn{3}{|l|}{ Tipo de Atención } \\
\hline Ambulatoria & 21 & 48.8 \\
\hline Hospitalización & 22 & 51.2 \\
\hline
\end{tabular}


[Tabla II. Prevalencias y sus intervalos de confianza (IC95\%) de los diagnósticos de enfermería de interés en niños que reciben tratamiento oncológico. Bucaramanga, Colombia.]

\begin{tabular}{clccc}
\hline $\mathbf{N}^{\mathbf{0}}$ & \multicolumn{1}{c}{ Diagnóstico de Enfermería } & $\mathbf{n = 4 3}$ & $\mathbf{\%}$ & $\mathbf{I C 9 5 \%}$ \\
\hline 1 & Protección Inefectiva & 35 & 81.4 & $66.6-91.6$ \\
2 & Riesgo de Infección & 34 & 79.1 & $64.0-90.0$ \\
3 & Riesgo de Lesión & 31 & 72.1 & $56.3-84.7$ \\
4 & Náusea & 30 & 69.8 & $53.9-82.8$ \\
5 & Trastorno de la Imagen Corporal & 22 & 51.2 & $35.5-66.7$ \\
6 & Fatiga & 19 & 44.2 & $29.1-60.1$ \\
7 & Intolerancia a la Actividad & 17 & 39.5 & $25.0-55.6$ \\
8 & Déficit de Actividades Recreativas & 17 & 39.5 & $25.0-55.6$ \\
9 & Deterioro de la Dentición & 13 & 30.2 & $17.2-46.1$ \\
10 & Temor & 12 & 27.9 & $15.3-43.7$ \\
11 & Deterioro de la Mucosa Oral & 11 & 25.6 & $13.5-41.2$ \\
12 & Dolor Agudo & 11 & 25.6 & $13.5-41.2$ \\
13 & Deterioro de la Deambulación & 9 & 20.9 & $10.0-36.0$ \\
14 & Desequilibrio Nutricional por Defecto & 9 & 20.9 & $10.0-36.0$ \\
15 & Deterioro de la Interacción Social & 8 & 18.6 & $8.4-33.4$ \\
16 & Insomnio & 6 & 14.0 & $5.3-27.9$ \\
17 & Hipertermia & 6 & 14.0 & $5.3-27.9$ \\
18 & Deterioro de la Integridad Cutánea & 6 & 14.0 & $5.3-27.9$ \\
19 & Aislamiento Social & 5 & 11.6 & $3.9-25.1$ \\
20 & Desesperanza & 5 & 11.6 & $3.9-25.1$ \\
21 & Baja Autoestima Situacional & 5 & 11.6 & $3.9-25.1$ \\
22 & Estreñimiento & 4 & 9.3 & $2.6-22.1$ \\
23 & Retraso en el Crecimiento y Desarrollo & 3 & 7.0 & $1.5-19.1$ \\
24 & Diarrea & 2 & 4.7 & $0.6-15.8$ \\
25 & Riesgo de Estreñimiento & 2 & 4.7 & $0.6-15.8$ \\
26 & Deterioro de la Eliminación Urinaria & 2 & 4.7 & $0.6-15.8$ \\
27 & Dolor Crónico & 0 & 0 & $0-8.2$ \\
\hline
\end{tabular}

A partir de los datos de prevalencia de la Tabla II se pudo obtener el número de diagnósticos de enfermería en cada niño. La Figura I presenta estos resultados, de donde entre otros datos se puede afirmar que cerca del $5 \%$ de los niños $(n=2)$ presentaron el menor número de diagnósticos, que corresponden a 2 dos diagnósticos y el $7 \%$ de los niños $(n=3)$ presentaron el mayor número de diagnósticos, que corresponden a 21 diagnósticos; el promedio de número de diagnósticos de enfermería por paciente fue de 7.7 (DE = 5.1) 
[Figura I. Frecuencia del número de diagnósticos de enfermería presentes en niños que reciben tratamiento oncológico. Bucaramanga, Colombia.]

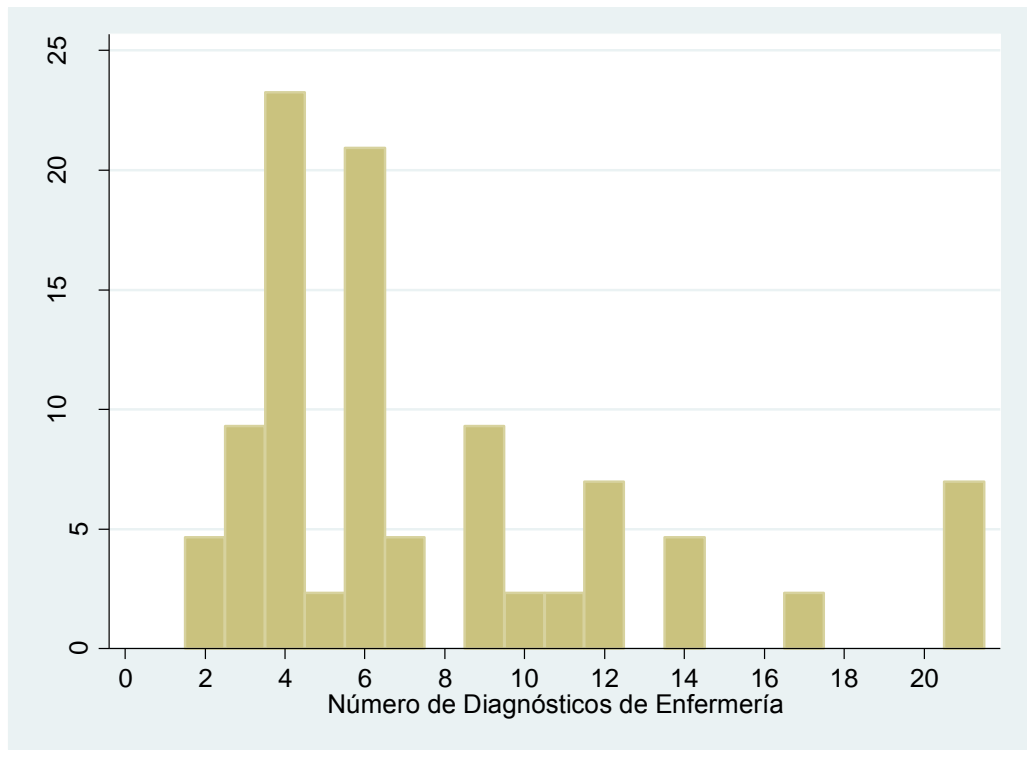

\section{DISCUSIÓN}

Los diagnósticos de enfermería hallados con mayor prevalencia: protección inefectiva, riesgo de infección, riesgo de lesión, náuseas y trastorno de la imagen corporal, están relacionados con los efectos tóxicos que ocasiona el tratamiento oncológico. Lo anterior, guarda relación con varios estudios sobre los efectos secundarios del tratamiento oncológico ${ }^{(15)(16)}$, los cuales muestran que en el tratamiento del enfermo con cáncer pueden surgir efectos tóxicos secundarios a la terapéutica utilizada bien sea radioterapia o quimioterapia. Dichos efectos pueden comprometer diversos tejidos u órganos, siendo los más frecuentes a nivel gastrointestinal como las náuseas y vómitos que se constituyen en los más temidos por los pacientes, dependiendo principalmente del tipo y dosis del quimioterapéutico o del lugar y dosis de la radioterapia.

Por otro lado, dentro de los efectos hematológicos, la neutropenia es con frecuencia un efecto tóxico secundario al tratamiento quimioterapéutico del paciente oncológico y se define como la disminución del recuento absoluto de neutrófilos $(<1500 / \mathrm{mm} 3)$. La principal complicación que se puede producir en el paciente neutropénico es la infección, que puede llegar a ser mortal. La severidad de la infección es inversamente proporcional al recuento de neutrófilos. Además, la anemia puede ser una de las manifestaciones del paciente con neoplasias hematológica, su prevalencia oscila entre el $30-90 \%$. También puede ser un efecto tóxico secundario al tratamiento oncológico, tanto de la quimioterapia como de la radioterapia.

Uno de los diagnósticos prevalentes fue trastorno de la imagen corporal, diagnóstico que se relaciona con la pérdida de cabello, que es uno de los efectos secundarios más frecuentes del tratamiento oncológico. Se trata de un efecto tóxico normalmente reversible pero con gran repercusión psicológica, que afecta principalmente a la cabeza pero puede aparecer en cualquier parte del cuerpo ${ }^{(16)}$.

La importancia de la imagen corporal en personas que reciben tratamiento oncológico, se evidencia en la revisión hecha por Dougherty ${ }^{(17)}$, donde afirma que los diagnósticos de enfermería claves relacionados con la alopecia inducida por la quimioteraía son el trastorno 
de la imagen corporal y duelo. El primero de estos fue el quinto mas prevalente para nuestro estudio presentándose en un 51.16\% y aunque el diagnóstico de duelo no fue tenido en cuenta, se puede resaltar que se encontraron diagnósticos relacionados como el temor con un $27.91 \%$ y desesperanza con un $11.63 \%$.

En un estudio realizado en adultos que recibían tratamiento oncológico ${ }^{(18)}$, encontraron una alta prevalencia de diagnósticos de enfermería positivos (disposición para mejorar el régimen terapéutico, disposición para mejorar la comunicación y conductas generadoras de salud); además, se presentaron diagnósticos para mejorar el afrontamiento, la espiritualidad y la religiosidad, los cuales en la población infantil no son prevalentes debido a que los niños manejan otras perspectivas con respecto a su patología, por lo cual sus diagnósticos se centran principalmente en el confort, el estado de salud y comparten las mismas necesidades de cuidado por parte de enfermería, también porque entre los diagnósticos de enfermería seleccionados para el presente estudio no se encontraban diagnósticos positivos.

Es importante resaltar que los hallazgos del presente estudio en niños demuestran las diferencias existentes en lo concerniente a las prevalencias y tipos de respuestas humanas en personas que reciben tratamiento oncológico cuando se compara con los resultados en estudios en adultos ${ }^{(18,19)}$. Asimismo estos resultados y diferencias facilitan la compresión de las necesidades, emociones y sentimientos de los niños en tratamiento para el cáncer, para que enfermería pueda brindar un cuidado efectivo, holístico y oportuno, enmarcado en una práctica basada en la evidencia.

Por otra parte, una de las ventajas del presente estudio fue el hecho de recolectar la información en dos instituciones diferentes de oncología de la ciudad, porque permitió una mayor captación de casos. Lo anterior, da la posibilidad para realizar una próxima investigación en la que se comparen los resultados de las dos instituciones de salud para así determinar las posibles diferencias o relaciones que existan entre las mismas.

Este tipo de estudios permiten al equipo de salud, en especial a enfermería, programar las necesidades de personal, tiempo del mismo, así como sus costos, para realizar las valoraciones, diagnósticos, intervenciones y evaluación de resultados ${ }^{(20,21)}$, lo cual redundará en un mejor cuidado de estas personas.

\section{CONCLUSIÓN}

El aporte de esta investigación para enfermería en los servicios de oncología pediátrica consiste principalmente en priorizar los cuidados específicos que se deben brindar a esta población, mediante un plan de cuidados estandarizado teniendo en cuenta la prevalencia de los diagnósticos encontrados y las necesidades reales de los niños. Esta investigación se convierte en una herramienta indispensable tanto en la asistencia como en la docencia puesto que la evidencia orienta el cuidado a seguir.

\section{BIBLIOGRAFÍA}

1. Ibañez-Alfonso LE, Sarmiento de Peña LV. El proceso de enfermería. Primera Ed. Bucaramanga: Universidad Industrial de Santander; 2003.

2. Wooldridge JB, Brown OF, Herman J. Nursing diagnosis: the central theme in nursing knowledge. Nursing Diagnosis: ND: The Official Journal of the North American Nursing Diagnosis Association. 1993 Jun;4(2):50-5. 
3. Hootman J. Nursing diagnosis--a language of nursing; a language for powerful communication. The Journal of School Nursing: The Official Publication of the National Association of School Nurses. 1996 Dec;12(4):19-23.

4. Barraso P, Granados Matute A, Fraile Rodríguez P. ACTUACIÓN DE ENFERMERÍA ANTE EL NIÑO CON CÁNCER: PLAN DE CUIDADOS. Enfermería Global. 2007 Nov;6(11). Disponible en: http://revistas.um.es/eglobal/article/view/429

5. Pizzo PA, Poplack DG. Principles and practice of pediatric oncology. Fourth. Philadelphia: Lippincott Williams \& Wilkins; 2001.

6. Departamento Administrativo Nacional de Estadística (DANE). Defunciones. Bógota, D.C.: 1999. Disponible en: http://www.dane.gov.co

7. Maureen A. Child Health 2000: 2nd World Congress and Exposition. Vancouver, Canada, May 30-June 3, 1995. Concurrent conferences on hematology--oncology: Challenges in childhood cancer and blood diseases. Journal of pediatric hematology/oncology. 1996 Mar;18(1):3-9.

8. Courtens A, Abu-Saad HH. Nursing diagnoses in patients with leukemia. International Journal of Nursing Terminologies and Classifications. 1998 Jun;9(2):49-61.

9. Palma C, Sepúlveda F. Atención de enfermería en el niño con cáncer. Revista Pediatría Electrónica. 2005;2(2). Disponible en: http://www.revistapediatria.cl/vol2num2/10.htm

10. Carrión PG, Muñoz AC. Experiencias y necesidades percibidas por los niños y adolescentes con cáncer y por sus familias. Nure Investigación. 2005;16. Disponible en: http://www.fuden.es/originales detalle.cfm?ID ORIGINAL=86\&ID ORIGINAL INI=1

11. NANDA International. Diagnósticos enfermeros: definiciones y clasificación 2007-2008. Madrid, España.: Elsevier España; 2008.

12. Carpenito-Moyet LJ. Nursing diagnosis: Application to clinical practice. 11th ed. Philadelphia: Lippincott Williams \& Wilkins; 2006.

13. CDC. Epi Info (programa de ordenador) versión 6.04 d. Atlanta (Georgia): Epidemiología en ordenadores. 2001.

14. StataCorp L. Stata Statistical Software: Release 10. Statacorp, College Station, TX USA. 2009.

15. Bryant R. Managing side effects of childhood cancer treatment. Journal of Pediatric Nursing. 2003 Apr;18(2):113-25.

16. Alfonsín FL, Campelo MRG, Pato GC, Calvo OF. Efectos secundarios del tratamiento oncológico. Cadernos de Atención Primaria. 2006;13:15-21.

17. Dougherty L. Using Nursing Diagnoses in Prevention and Management of ChemotherapyInduced Alopecia in the Cancer Patient. International Journal of Nursing Terminologies and Classifications. 2007;18(4):142-9.

18. Cáceres-Manrique F, Puerto-Pedraza HM. Prevalencia de diagnósticos de Enfermería en personas en tratamiento oncológico. Investigación y Educación en Enfermería. 2009;26(2):212-7.

19. Gallegos-Alvarado M, Parra-Domínguez ML. Diagnósticos de enfermería identificados en pacientes con cáncer. Revista CONAMED. 2008;13(S1):12-6.

20. Haugsdal CS, Scherb CA. Using the nursing interventions classification to describe the work of the nurse practitioner. Journal of the American Academy of Nurse Practitioners. 2003 Feb;15(2):87-94.

21. Dochterman JM, Bulechek G, Head B, Ahrens D, Androwich I, Clarke M, et al. Determining cost of nursing interventions: A beginning. Nursing Economics. $2001 ; 19(4): 146-60$. 
